\title{
Chemokine (C-C motif) ligand 21/C-C chemokine receptor type 7 triggers migration and invasion of human lung cancer cells by epithelial-mesenchymal transition via the extracellular signal-regulated kinase signaling pathway
}

\author{
GUANGXIN ZHONG ${ }^{1}$, LU CHEN ${ }^{1}$, RUIHONG YIN $^{2}$, YAN QU ${ }^{1}$, YONGXING BAO ${ }^{1}$, QIONG XIAO $^{3}$, \\ ZHAOLIN ZHANG ${ }^{4}$, YAQIAN SHEN ${ }^{1},{\text { CAILING } \text { LI }^{1}, \text { YUN XU }^{5} \text {, ZHIGENG ZOU }}^{6}$ and HUA TIAN ${ }^{1}$
}

\footnotetext{
${ }^{1}$ Institute of Anatomy and Histology and Embryology, School of Medicine, Shandong University, Jinan, Shandong 250012;

${ }^{2}$ Department of Internal Medicine, Jinan First People's Hospital, Jinan, Shandong 250000; ${ }^{3}$ Blood Center of General Hospital of Jinan Military Region, Jinan, Shandong 250031; ${ }^{4}$ Department of Special Examination, Penglai People's Hospital,

Penglai, Shandong 265600; ${ }^{5}$ Department of Anatomy, Jining Medical University, Jining, Shandong 272000;

${ }^{6}$ Cancer Treatment Center, Shandong Provincial Hospital, Shandong University, Jinan, Shandong 250021, P.R. China
}

Received January 12, 2016; Accepted February 14, 2017

DOI: $10.3892 / \mathrm{mmr} .2017 .6534$

\begin{abstract}
C-C chemokine receptor type 7 (CCR7) has been implicated in lymph node metastasis of various cancers. Previous studies have revealed that epithelial-mesenchymal transition (EMT) is involved in the chemotactic process mediated by CCR7 and its ligands in various types of carcinoma. However, the underlying mechanism of this process remains to be fully elucidated. The present study investigated whether chemokine (C-C motif) ligand 21 (CCL21)/CCR7 may activate EMT of lung cancer cells and their associated signaling pathways. A549 and H520 lung cancer cell lines were examined in vitro in the present study. The results indicated that A549 and H520 expressed CCR7, but reduced levels of CCL21. Following stimulation of lung cancer cell lines with CCL21, the expression of the epithelial marker E-cadherin was downregulated, and the mesenchymal markers Vimentin/Slug and extracellular signal-regulated kinase (ERK) were upregulated. In addition, the ERK inhibitor PD98059 may inhibit EMT caused by CCL21, and decreased cell migration and invasion initiated by CCL21. Furthermore, lung adenocarcinoma tissues from 50 patients
\end{abstract}

Correspondence to: Dr Zhigeng Zou, Cancer Treatment Center, Shandong Provincial Hospital, Shandong University, 324 Jingwu Weiqi Road, Jinan, Shandong 250021, P.R. China

E-mail: 88281986@qq.com

Professor Hua Tian, Institute of Anatomy and Histology and Embryology, School of Medicine, Shandong University, 44 Wenhua Xi Road, Jinan, Shandong 250012, P.R. China

E-mail: sduth@163.com

Key words: C-C chemokine receptor type 7, chemokine (C-C motif) ligand 21, epithelial-mesenchymal transition, extracellular signal-regulated kinase, lymph node metastasis, lung cancer who underwent lung cancer operations were investigated by immunohistochemistry. The findings revealed that CCR7, Slug and Vimentin were highly expressed in lung carcinoma tissues, and were significantly associated with lymph node metastasis and clinical pathological stages, respectively. CCR7 expression was correlated positively with expression levels of Slug and Vimentin. CCL21 was expressed positively in the endothelium of lymphatic vessels adjacent to cancer cells, and weakly in lung cancer cells. Collectively, these results demonstrated that CCL21/CCR7 may activate EMT in lung cancer cells via the ERK1/2 signaling pathway. The current study provides evidence that a close interaction exists between CCL21/CCR7chemotaxis and EMT procedures in lung cancer metastasis, providing a basis for the development of therapeutic targets.

\section{Introduction}

Epithelial-mesenchymal transition (EMT) and chemotaxis are critical processes in cancer metastasis (1-3), and their interaction in cancer metastasis has been investigated in recent years. Cancer cells acquire the capability to detach and migrate away from primary tumors by EMT, involving loss of an epithelial phenotype, including E-cadherin, and gain of a mesenchymal phenotype, including Vimentin and Slug $(1,2)$. Chemokine and receptor interactions guide cancer cells to migrate to distance favorite organs or lymph nodes (3).

Previous studies have identified that certain chemokine receptors, including $\mathrm{C}-\mathrm{X}-\mathrm{C}$ chemokine receptor type 4 (CXCR4) and C-C chemokine receptor type 7 (CCR7), have been detected in a variety of cancer cells and were driven by their ligands to targeted metastasis of cancers $(3,4)$. CCR7 is shared by its ligands chemokine (C-C motif) ligand (CCL) 19 or CCL21, which are produced specifically in secondary lymphatic tissues, including lymph nodes, Peyer's patches and splenic follicles (5-8). Therefore, CCR7 is considered to contribute to 
lymph node metastasis of cancers $(9,10)$. Many growth factors, including transforming growth factor (TGF)- $\beta$, basic fibroblast growth factor and platelet-derived growth factor have been identified as EMT inducers, and the molecular mechanisms underlying promotion of cancer cell invasion have been investigated (1). Notably, previous studies have revealed that CXCR4 (11,12), CCR2 (13) and CCR6 (14) binding with their chemokines may induce EMT in cancer cells to stimulate cancer cell mobility. Recently, CCL21/CCR7 were demonstrated to activate EMT in human breast cancer cells (15), and subsequently, other studies reported that CCL21/CCR7 may activate the EMT process in gastric cancer cells (16).

Lung carcinoma is one of the most lethal malignancies with poor prognosis due to its ability to metastasize, causing $\sim 84,590$ mortalities in men and 71,280 mortalities in women in the United States in 2016 (17). However, the role of CCR7 and its ligands in EMT of human lung cancer cells remains to be fully clarified. The present study investigated the capacity of CCL21/CCR7 for stimulating EMT, and associated extracellular signal-regulated kinase (ERK) 1/2 signaling in lung cancer.

\section{Materials and methods}

Cell culture and reagents. The NCI-H157, A549, 973 and NCI-H520 human lung carcinoma cell lines were obtained from the American Type Culture Collection (Manassas, VA, USA). All cells were cultured in RPMI-1640 medium (Gibco; Thermo Fisher Scientific, Inc., Waltham, MA, USA) supplemented with $10 \%$ fetal bovine serum (FBS; Gibco; Thermo Fisher Scientific, Inc.) at $37^{\circ} \mathrm{C}$ in $5 \% \mathrm{CO}_{2}$. Protein expression levels of CCR7 and CCL21 in these cells were detected by western blot analysis, and the results indicated that CCR7 expression levels were differential in A549 and H520 cells; therefore, they were selected for further analysis. A549 and NCI-H520 cell lines were treated with or without CCL21 (cat. no. 300-35; PeproTech, Rocky Hill, NJ, USA) at designed concentrations and times. PD98059 (cat. no. 9900; Cell Signaling Technology, Danvers, MA, USA), a selective inhibitor of mitogen activated protein kinase kinase (MEK), was used to estimate disruption to the ERK signaling pathway, which is downstream of MEK.

RNA extraction and reverse transcription-quantitative polymerase chain reaction $(R T-q P C R)$. Total RNA was isolated using TRIzol ${ }^{\circledR}$ (Invitrogen; Thermo Fisher Scientific, Inc.) according to the manufacturer's protocol. cDNA was reverse transcribed from the mRNA using a Revert Aid First Strand cDNA Synthesis kit (cat. no. K01622; Thermo Fisher Scientific, Inc.) following the manufacturer's protocol. mRNA of EMT biomarkers including E-cadherin, Vimentin and Slug, as well as GAPDH were detected by RT-qPCR using Maxima SYBR $^{\circledR}$-Green qPCR Master Mix (cat. no. K0252; Thermo Fisher Scientific, Inc.). The data were obtained and analyzed using a Mx4000 Multiplex Quantitative PCR system (version, 3.0; Stratagene; Agilent Technologies, Inc., Santa Clara, CA, USA). Relative mRNA expression was calculated by the $2^{-\Delta \Delta C q}$ method (18). All values were normalized to an endogenous GADPH control. The primers (BioSune, Shanghai, China) used in RT-qPCR were as follows: Forward, 5'-CTGAGAACG
AGGCTAACG-3' and reverse, 5'-GTCCACCATCATCAT TCAATAT-3' for E-cadherin; forward, 5'-TCCGCCAGCAGT ATGAAAG-3' and reverse, 5'-TGGGTGTCAACCAGAGGA AG-3' for Vimentin; forward, 5'-CAGCTCAGGAGCATA CAG-3' and reverse, 5'-GAGGAGGTGTCAGATGGA-3' for Slug; and forward, 5'-GCCGCATCTTCTTTTGCGTCGC-3' and reverse, 5'-TCCCGTTCTCAGCCTTGACGGT-3' for GAPDH. The PCR conditions were: Initial denaturation at $95^{\circ} \mathrm{C}$ for $30 \mathrm{sec}$ followed by 40 cycles of denaturation at $95^{\circ} \mathrm{C}$ for $5 \mathrm{sec}$, and annealing at $60^{\circ} \mathrm{C}$ for $30 \mathrm{sec}$. Experiments were performed in triplicate.

Western blot analysis. The total protein was extracted from the cultured cells using the Beyotime lysis buffer containing radioimmunoprecipitation buffer and a protease inhibitor cocktail (Beyotime Institute of Biotechnology, Haimen, China) followed by centrifugation in $12,000 \mathrm{x}$ g at $4^{\circ} \mathrm{C}$ for $30 \mathrm{~min}$. A total of 50-60 $\mu \mathrm{g}$ of protein lysates were separated by $10 \%$ SDS-PAGE and subsequently transferred onto polyvinylidene fluoride membranes (PVDF; EMD Millipore, Billerica, MA, USA), followed by blocking with $5 \%$ bovine serum albumin (Amresco, LLC, Solon, OH, USA) in TBS with Tween-20 for $2 \mathrm{~h}$. The PVDF membranes were incubated with the following primary antibodies: Anti- $\beta$-actin (dilution, 1:2,000; cat. no. 8457P; Cell Signaling Technology, Inc.), anti-CCR7 (dilution, 1:5,000; cat. no. ab32527; Abcam, Cambridge, UK), anti-CCL21 (dilution, 1:400; cat. no. sc-5808; Santa Cruz Biotechnology, Inc., Dallas, TX, USA), anti-Slug (dilution, 1:400; cat. no. sc-166476; Santa Cruz Biotechnology, Inc.), anti-E-cadherin (dilution, 1:1,000; no. ab133597; Abcam), anti-Vimentin (dilution, 1:1,000; cat. no. 5741P; Cell Signaling Technology, Inc.), anti-phosphorylated (p)-ERK1/2 (dilution 1:1,000; cat. no. 4370; Cell Signaling Technology, Inc.) and anti-ERK1/2 (dilution, 1:1,000; cat. no. 4695; Cell Signaling Technology, Inc.) overnight at $4^{\circ} \mathrm{C}$. Horseradish peroxidase-conjugated secondary antibodies, including goat anti-mouse IgG (dilution, 1:10,000; cat. no. ZB-5305; ZSGB-Bio, Beijing, China), goat anti-rabbit IgG (dilution, 1:10,000; cat. no. ZB-5301; ZSGB-Bio) and rabbit anti-goat IgG (dilution, 1:10,000; cat. no. ZB-2306; ZSGB-Bio) were used at room temperature for $1 \mathrm{~h}$ for the detection of immunoreactive proteins by a luminochemiluminescence reagent (Santa Cruz Biotechnology, Inc.). The semi-quantitative analyses of data were carried out using ImageJ (version, 2.1; Windows 7; National Institutes of Health, Bethesda, MD, USA). The experiment was performed three times.

Wound healing assay. A549 and NCI-H520 cells (3x10 $)$ were seeded in 6-well culture dishes and cultured in RPMI-1640 medium (Gibco; Thermo Fisher Scientific, Inc.) containing 10\% FBS (Gibco; Thermo Fisher Scientific, Inc.), incubated with or without $50 \mu \mathrm{M}$ PD98059 (cat. no. 9900; Cell Signaling Technology, Inc.) at $37^{\circ} \mathrm{C}$ for $1 \mathrm{~h}$, and subsequently treated with 100 ng/ml CCL21 (cat. no. 300-35; PeproTech). When cells reached confluence, a scratch in the central area was established by a sterile pipette tip, followed by washing with PBS to remove cell debris and replenishing with fresh medium. Following incubation for $48 \mathrm{~h}$, the migrated cells in the wound space were counted under a phase-contrast light microscope. The experiment was repeated three times. 
Table I. Correlation of CCR7/Vimentin/Slug expression with clinical pathological parameters in lung carcinoma tissues.

\begin{tabular}{|c|c|c|c|c|c|c|c|c|c|c|}
\hline \multirow[b]{2}{*}{ Parameter } & \multirow[b]{2}{*}{$\mathrm{n}$} & \multicolumn{3}{|c|}{ CCR7 } & \multicolumn{3}{|c|}{ Slug } & \multicolumn{3}{|c|}{ Vimentin } \\
\hline & & Low & High & $\mathrm{P}$ & Low & High & $\mathrm{P}$ & Low & High & $\mathrm{P}$ \\
\hline Age & & & & 0.887 & & & 0.623 & & & 0.598 \\
\hline$\leq 50$ & 26 & 5 & 21 & & 5 & 21 & & 10 & 16 & \\
\hline$>50$ & 24 & 5 & 19 & & 6 & 18 & & 11 & 13 & \\
\hline Sex & & & & 0.254 & & & 0.793 & & & 0.917 \\
\hline Female & 29 & 4 & 24 & & 6 & 23 & & 12 & 17 & \\
\hline Male & 21 & 6 & 16 & & 5 & 16 & & 9 & 12 & \\
\hline $\begin{array}{l}\text { Lymph node } \\
\text { status }\end{array}$ & & & & $0.010^{\mathrm{a}}$ & & & $0.030^{\mathrm{a}}$ & & & $0.030^{\mathrm{a}}$ \\
\hline Positive & 28 & 2 & 26 & & 3 & 25 & & 8 & 20 & \\
\hline Negative & 22 & 8 & 14 & & 8 & 14 & & 13 & 9 & \\
\hline Stage & & & & 0.063 & & & 0.055 & & & 0.235 \\
\hline I & 9 & 2 & 7 & & 3 & 6 & & 5 & 4 & \\
\hline II & 4 & 6 & 8 & & 6 & 8 & & 9 & 5 & \\
\hline III & 12 & 1 & 11 & & 1 & 11 & & 4 & 8 & \\
\hline IV & 15 & 1 & 14 & & 1 & 14 & & 3 & 12 & \\
\hline
\end{tabular}

${ }^{\text {aP }}<0.05$ vs. lymph node status. CCR7, C-C chemokine receptor type 7.

Matrigel invasion assay. A Transwell chamber system (24 wells; 8 mm pore size; BD Biosciences, Franklin Lakes, NJ, USA) was used in A549 and NCI-H520 cell lines. The Transwell filters (polycarbonate membrane) were pre-coated with Matrigel (cat. no. 356234; BD Biosciences). Tumor cells were pre-incubated with or without $50 \mu \mathrm{M}$ PD98059 (cat. no. 9900; Cell Signaling Technology, Inc.) at $37^{\circ} \mathrm{C}$ for $1 \mathrm{~h}$, following which $2 \times 10^{5}$ cells suspended in RPMI-1640 medium (Gibco; Thermo Fisher Scientific, Inc.) containing 10\% FBS (Gibco; Thermo Fisher Scientific, Inc.) and 100 ng/ml CCL21 (cat. no. 300-35; PeproTech) were loaded on Matrigel in the upper chamber. RPMI-1640 medium (Gibco; Thermo Fisher Scientific, Inc.) containing 10\% FBS (Gibco; Thermo Fisher Scientific, Inc.) was added to the lower chamber. Following $48 \mathrm{~h}$ incubation at $37^{\circ} \mathrm{C}$, noninvasive cells on the upper surface of Matrigel membrane (cat. no. 356234; BD Biosciences) were removed with a cotton swab. The invading cells on the underside of the Matrigel membrane (cat. no. 356234; BD Biosciences) were fixed with methanol for $30 \mathrm{~min}$ at $37^{\circ} \mathrm{C}$, stained with $1 \%$ crystal violet for $30 \mathrm{~min}$ at $37^{\circ} \mathrm{C}$ and counted in at least five random separate fields under a light microscope. The assay was performed in triplicate.

Immunohistochemistry. Lung adenocarcinoma tissues were selected from 50 patients ( 29 females and 21 males) who underwent radical resection of lung cancer and had been clinically and histopathologically diagnosed in Shandong Tumor Hospital (Jinan, China). The tissues were blocked by $10 \%$ formalin and embedded in paraffin. No patients had received chemotherapy or radiation therapy prior to surgery. The TNM stage system (19) was applied to classify the specimens. Clinical pathological information was obtained from patient records in hospital and is summarized in Table I. All samples used in the study were anonymized and informed consent was received from all patients. The procedure in the present study was approved by the Medical Ethical Committee of Shandong University (Jinan, China).

The paraffin-embedded tissues were cut into $\sim 5 \mu \mathrm{m}$-thick sections, and immunohistochemical staining was performed according to the manufacturer's protocol. Tissue sections were de-waxed in xylene and rehydrated in a graded alcohol series. Antigen retrieval was conducted. The endogenous peroxidase of tissues was inactivated afterwards. Tissue slides were incubated with the following primary antibodies at $4^{\circ} \mathrm{C}$ overnight: anti-Vimentin (dilution, 1:200; cat. no. 5741P; Cell Signaling Technology, Inc.), anti-Slug (dilution, 1:200; cat. no. sc-166476; Santa Cruz Biotechnology, Inc.), anti-CCL21 (dilution, 1:200; cat. no. sc-5808; Santa Cruz Biotechnology, Inc.) and anti-CCR7 (dilution, 1:500; cat. no. ab32527; Abcam). PBS was used as negative control. Using the EnVision System (Dako; Agilent Technologies, Inc.) primary antibodies were visualized, and nuclei were counterstained by hematoxylin.

Immunostaining scoring for staining intensity and positive percentage in five high-power fields was performed according to a previous protocol under a light microscope (magnification, $\mathrm{x} 400)$ (11). The intensity score was obtained by background staining as was graded as follows: None, 0; mild, 1 ; moderate, 2 ; intense, 3 . The percentage of positive tumor cells were assessed according to the following patterns: $0 \%$ cells, $0 ; 0-25 \%$ cells, $1 ; 25-50 \%$ cells, $2 ;>50 \%$ cells, 3 . The final score was obtained by multiplying the positive percentage score by the intensity score for each section, which ranged from 0 to 9 . If the final score was $<4$, the tissue sections were classified as negative or low expression. In 
contrast, if the score $\geq 4$, they were regarded as positive or high expression.

The same method was applied to assess the expression of CCR7, Vimentin and Slug in interstitial stromal cells.

Statistical analysis. Data are presented as the mean \pm standard deviation of three independent experiments. A chi-squared test was used for evaluating the association between CCR7 and EMT biomarkers in human lung carcinoma and lymphoid tissues. Differences among groups were compared by one-way analysis of the variance and a Tukey post hoc test. $\mathrm{P}<0.05$ was considered to indicate a statistically significant difference. Statistical analysis was carried out using SPSS software (version, 18.0; SPSS Inc., Chicago, IL, USA).

\section{Results}

Differential expression of CCR7 and CCL21 in human lung cancer cells. To detect CCR7 and CCL21 expression in different lung cancer cell lines, western blot analysis was performed. CCR7 was expressed at different levels in H157, A549, 973 and H520 cell lines, appearing visibly increased in H520 and A549 cells (Fig. 1A); therefore, H520 and A549 cell lines were selected for the following assays. However, CCL21 was not detected in all cell lines (data not shown).

EMT phenotype expression is induced by CCL21 stimulation in lung cancer cells. To evaluate the roles of CCL21/CCR7 in activating EMT processes in human lung cancer cells, H520 and A549 cells were treated with or without $100 \mathrm{ng} / \mathrm{ml} \mathrm{CCL21}$ for $48 \mathrm{~h}$. The results of the western blot analysis demonstrated that CCL21 may promote EMT biomarker protein expression in lung cancer cells, including decrease of E-cadherin and increase of Vimentin and Slug (Fig. 1B).

ERK signaling is activated in response to CCL21 stimulation in lung cancer cells. To investigate whether the ERK signaling pathway may be activated by CCL21, H520 and A549 cells were treated with or without CCL21 in different concentrations $(0,50,100$ or $200 \mathrm{ng} / \mathrm{ml})$ for $48 \mathrm{~h}$, and the protein expression levels of p-ERK were detected by western blot analysis. The results demonstrated that ERK phosphorylation was positively increased in response to CCL21 in a dose-dependent manner (Fig. 1C).

Inhibition of ERK phosphorylation blocks EMT transformation in response to CCL21/CCR7 in lung cancer cells. To investigate the potential roles of ERK1/2 in CCL21/CCR7-mediated EMT activation in human lung cancer cells, PD98059, a selective inhibitor of MEK, was used to disrupt activation of its downstream target, ERK. H520 and A549 cells were pre-treated with or without $50 \mu \mathrm{M}$ PD98059 for $1 \mathrm{~h}$, followed by stimulation with or without $100 \mathrm{ng} / \mathrm{ml}$ CCL21 for $48 \mathrm{~h}$. Western blot analysis and RT-qPCR assays were performed. As presented in Fig. 2A, the western blot assay indicated that A549 and NCI-H520 cells presented a downregulation of E-cadherin and upregulation of Vimentin and Slug, as well as p-ERK, in response to CCL21 stimulation $(\mathrm{P}<0.01)$, but this phenotype transformation was abolished
A

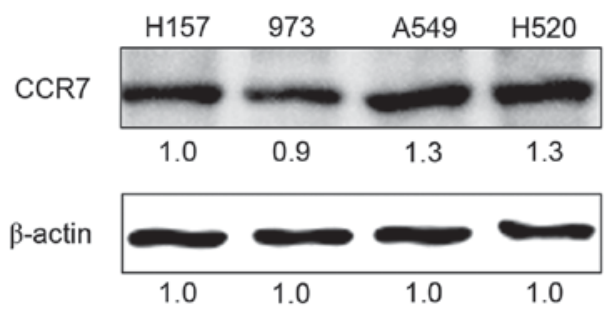

B

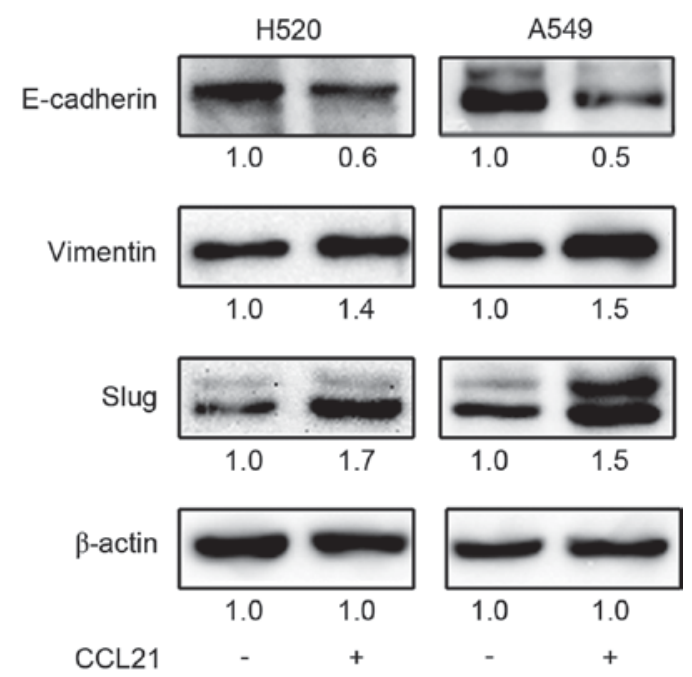

C

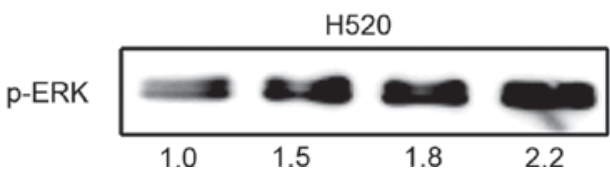

Figure 1. Detection of CCR7, CCL21, EMT biomarkers and p-ERK expression in response to CCL21 in lung cancer cells. Representative western blot images of (A) CCR7 (B) E-cadherin, Vimentin, Slug and (C) p-ERK protein expression levels. $\beta$-actin served as an internal loading control. CCR7, C-C chemokine receptor type 7; CCL21, chemokine (C-C motif) ligand 21; EMT, epithelial-mesenchymal transition; ERK, extracellular-regulated signal kinase; p, phosphorylated.

when the cells were pretreated with PD98059 $(\mathrm{P}<0.01)$. The results of RT-qPCR were consistent with that of the western blot (Fig. 2B).

ERK1/2 promotes migration of human lung cancer cells in response to CCL21/CCR7. The influence of ERK1/2 on cancer cell migration behavior was investigated using a wound-healing assay. H520 (Fig. 3A) and A549 (Fig. 3B) cells were cultured with or without PD98059, an inhibitor of MEK, followed by $100 \mathrm{ng} / \mathrm{ml} \mathrm{CCL} 21$ stimulation for $48 \mathrm{~h}$. The results revealed that there were more cancer cells migrating to the wound area in response to CCL21 stimulation, whereas this was markedly inhibited when the cells were pre-treated with PD98059 (P<0.01; Fig. 3C).

ERK1/2 increases invasion of human lung cancer cells in response to CCL21/CCR7. The influence of ERK1/2 on invasiveness of human lung cancer cells was tested using a Matrigel invasion assay. Following 
A

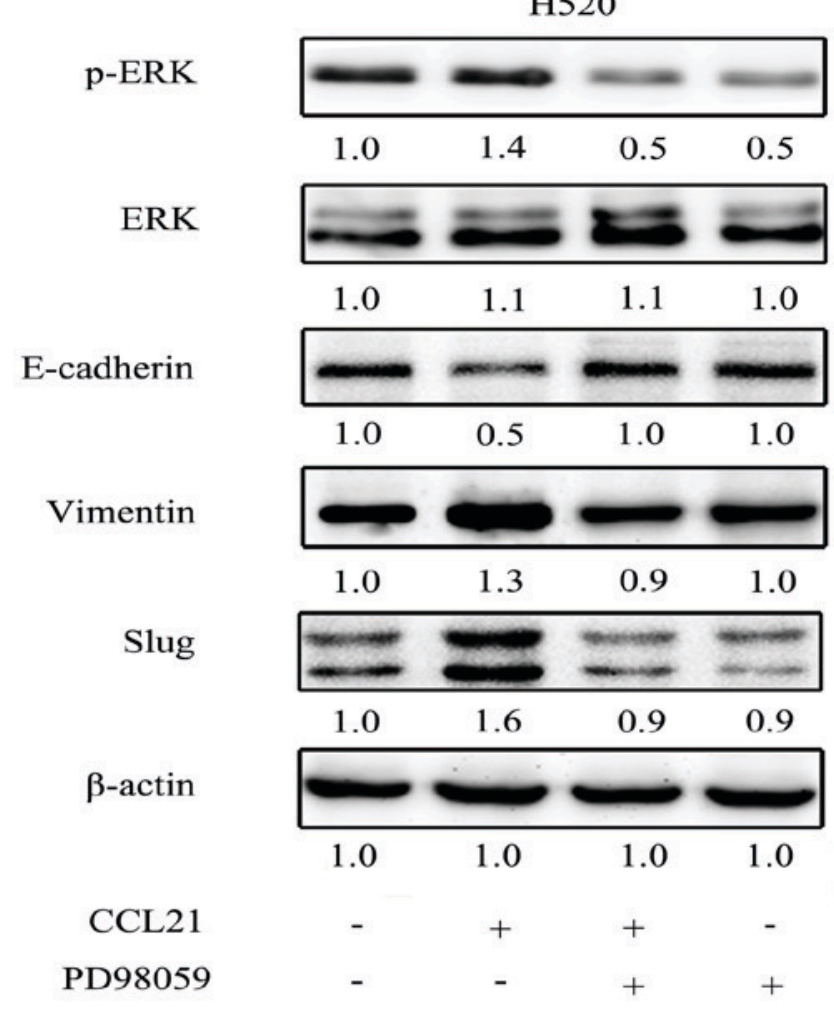

B
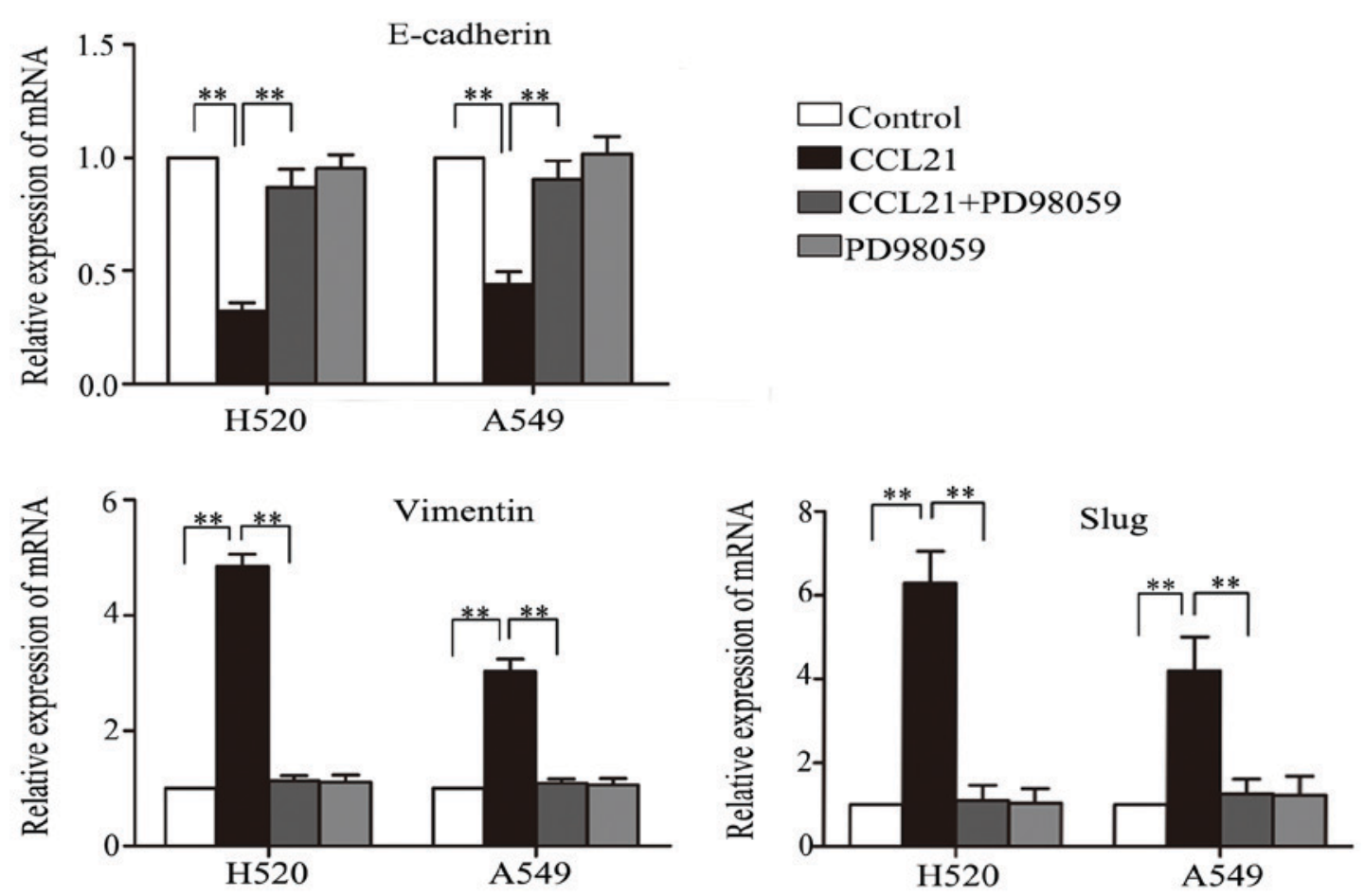

Figure 2. Roles of CCL21/CCR7 and p-ERK in EMT of lung cancer cells. (A) Western blot and (B) reverse transcription-quantitative polymerase chain reaction. Cancer cells presented a downregulation of E-cadherin and upregulation of Vimentin, Slug and p-ERK in response to CCL21 stimulation, when compared with the control group. These phenotype transformations in response to CCL21 stimulation above were abolished when the cells were pretreated with PD98059. Data are presented as the mean \pm standard deviation of three independent experiments. ${ }^{* *} \mathrm{P}<0.01$. CCL21, chemokine (C-C motif) ligand 21 ; CCR7, C-C chemokine receptor type 7; ERK, extracellular-regulated signal kinase; EMT, epithelial-mesenchymal transition.

pretreatment with PD98059 in H520 and A549 cells, followed by $100 \mathrm{ng} / \mathrm{ml} \mathrm{CCL} 21$ stimulation for $48 \mathrm{~h}$, the invaded cells travelling through the Matrigel were counted. The results indicated that the number of invasive cells in H520 (Fig. 4A) and A549 (Fig. 4B) samples were increased in response to CCL21 stimulation, whereas this was inhibited remarkably 


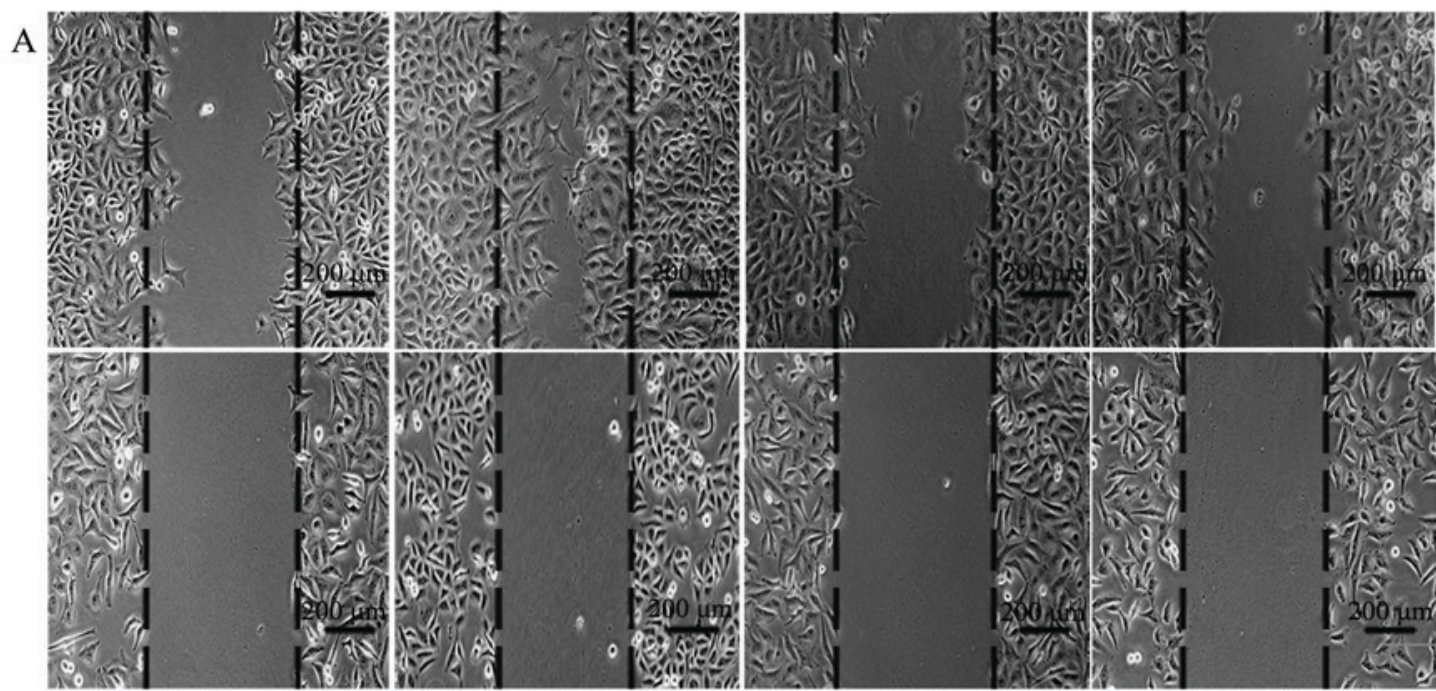

$48 \mathrm{~h}$

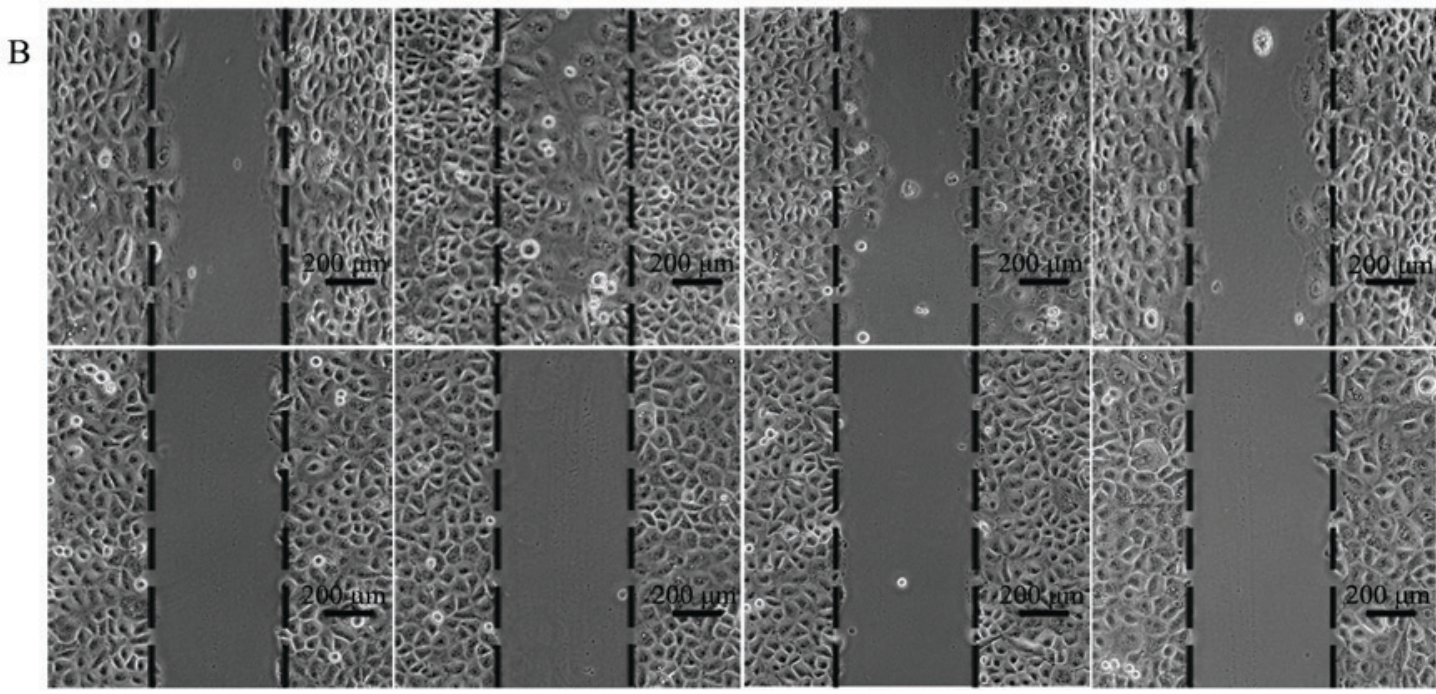

$48 \mathrm{~h}$

Oh

oh

CCL21
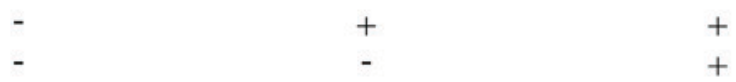

$+$

PD98059

C
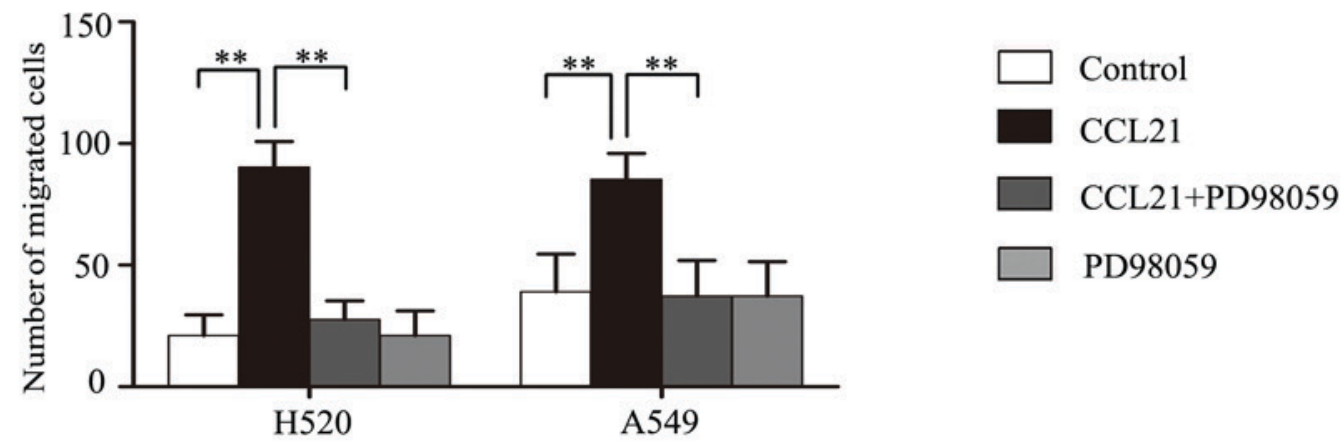

PD98059

Figure 3. Effect of ERK pathway in CCL21/CCR7 axis on migratory capacity of human lung cancer cells. (A) H520 and (B) A549 cells were subjected to wound-healing assay. (C) Quantification of the wound healing assay; more cancer cells migrated towards to wound area in response to CCL21 stimulation, when compared with the control group, whereas this was inhibited when the cells were pretreated with PD98059. Magnification, x200. Scale bar=200 $\mu \mathrm{m}$. ${ }^{* *} \mathrm{P}<0.01$. ERK, extracellular-regulated signal kinase; CCL21, chemokine (C-C motif) ligand 21; CCR7, C-C chemokine receptor type 7.

when the cells were pretreated with PD98059 $(\mathrm{P}<0.01$; Fig. 4C).

CCR7 expression correlates with clinicopathological parameters and Vimentin/Slug expression in human lung cancer tissues. Immunohistochemical staining demonstrated that CCR7 and Vimentin were expressed in the cytoplasm and/or plasma membranes of tumor cells, and Slug was expressed mainly in the nucleus and partially in the cytoplasm of cancer cells (Fig. 5). Furthermore, CCR7, Vimentin and 
A

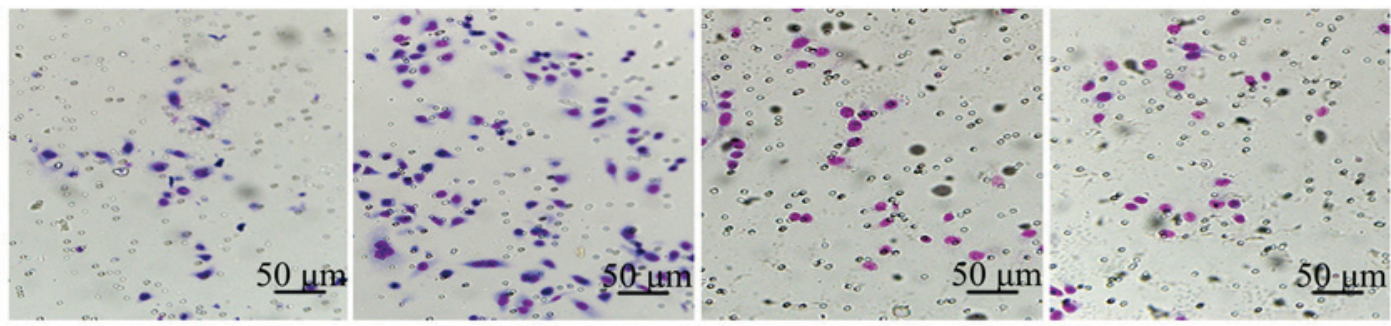

B

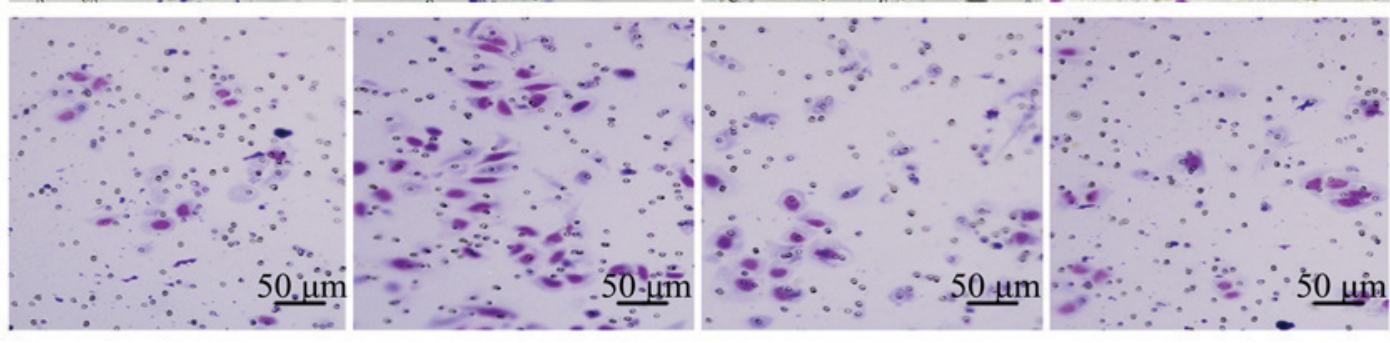

CCL21

PD98059

C

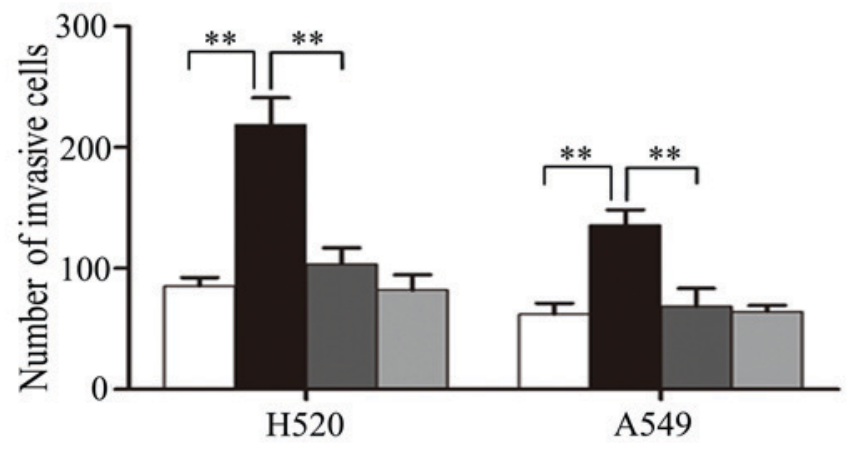

$\square$ Control

CCL21

$\square$ CCL21+PD98059

PD98059

Figure 4. Evaluation of invasive capacity in human lung cancer cells following ERK pathway inhibition. (A) H520 and (B) A549 cells were subjected to a Matrigel invasive assay. (C) The number of invasive cells treated with CCL21 was increased significantly in H520 and A549 cells, whereas this was significantly inhibited when the cells were pretreated with PD98059. Cells were imaged under a phase-contrast microscope. Magnification, 200 . Scale bar $=50 \mu \mathrm{m}$. ${ }^{* *} \mathrm{P}<0.01$. ERK, extracellular-regulated signal kinase; CCL21, chemokine (C-C motif) ligand 21.

Slug were additionally detected in 6 cases of interstitial stromal cells in cancer regions and/or in non-neoplastic tissue adjacent to carcinoma (data not shown), and the expression in interstitial stromal cells was assessed as above. The final score consists of tumor and interstitial stromal cells. CCR7, Slug and Vimentin were all highly expressed in lung carcinoma tissues with positive rates of $80 \%$ (40/50), $78 \%$ (39/50) and 58\% (29/50), respectively. Associations between expressions of CCR7/Slug/Vimentin and clinical parameters were analyzed and are presented in Table I. The expression levels of CCR7, Slug and Vimentin were significantly associated with lymph node metastasis $(\mathrm{P}=0.010, \mathrm{P}=0.030$ and $\mathrm{P}=0.030$, respectively). Additionally, CCR7 expression was correlated positively with expressions of Slug $(R=0.459$, $\mathrm{P}=0.001)$ and Vimentin $(R=0.385, \mathrm{P}=0.006)$. However, CCL21, the CCR7 ligand, was expressed mainly in the endothelium of lymphatic vessels adjacent to cancer cells (Fig. 5), but only weakly positive in cytoplasm of cancer cells in $34 \%(17 / 50)$ cases, which includes $20 \%(10 / 50)$ cases in the lymph node metastasis group and $14 \%(7 / 50)$ cases in the lymph node non-metastasis group. No significant differences were observed between these two groups.

\section{Discussion}

In the present study, the expression levels of CCR7 and CCL21 in various human lung cancer cell lines was analyzed. The results indicated that CCR7 was expressed in different cancer cell lines, and was most highly expressed in H520, a lung squamous cancer cell line, and A549, a lung non-small cell cancer cell line. However, CCL21 was not detected in any cell lines in vitro.

Based on the detection above, the expression levels of CCR7 and CCL21 in human lung cancer tissues were assessed by immunohistochemical staining. Similar to the findings in cancer cell lines, CCR7 was highly expressed in cancer cells in $80 \%$ of cancer tissues, and was associated with lymph node metastasis. Nevertheless, CCL21 was primarily expressed in the endothelium of lymphatic vessels surrounding the cancer foci, but hardly in cancer cells. Therefore, CCL21, released by 

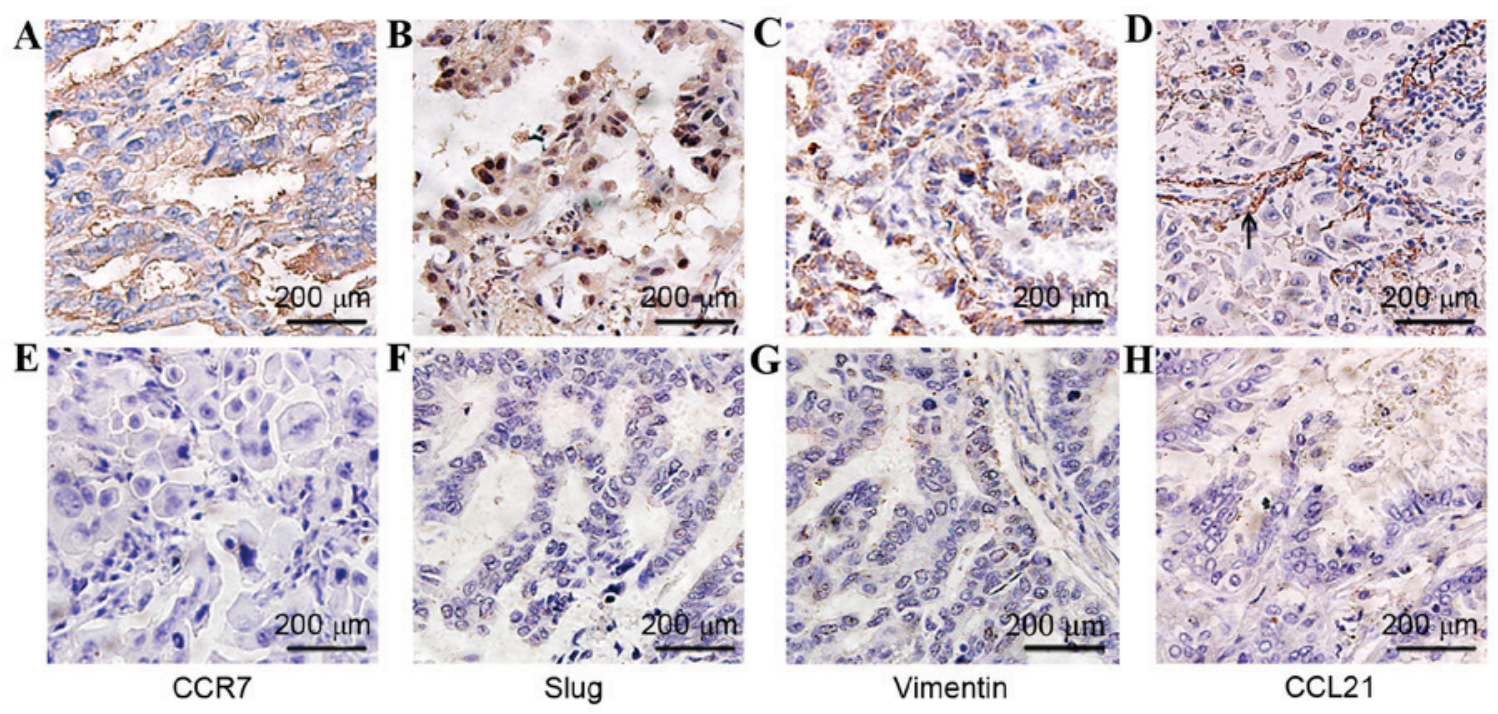

Figure 5. Immunohistochemical detection of CCR7, Slug, Vimentin and CCL21 expression in human lung cancer tissues. (A) CCR7 was strongly stained in the cytoplasm and/or plasma membrane of human lung cancer cells. (B) Slug was stained primarily in the nucleus and partly in the cytoplasm of cancer cells. (C) Vimentin were strongly stained in the cytoplasm and/or plasma membrane. (D) CCL21 was stained mainly in the lymphatic vessels around the cancer foci (as indicated by the black arrow). Negative control staining for (E) CCR7, (F) Slug, (G) Vimentin and (H) CCL21. Magnification, x200. Scale bar=200 $\mu$ m. CCR7, C-C chemokine receptor type 7; CCL21, chemokine (C-C motif) ligand 21.

lymphatic endothelium, may facilitate cancer cell migration to the lymphatic vessels. H520 and A549 cells were selected to evaluate whether CCL21/CCR7 activated EMT and the ERK signaling pathway in vitro.

The results demonstrated that CCL21/CCR7 effectively induced EMT via the ERK signaling pathway in the H520 and A549 lung cancer cell lines. Following treating with CCL21, the EMT phenotype was accelerated in H520 and A549 cells. The epithelial marker E-cadherin was downregulated, whereas the mesenchymal markers, Vimentin and Slug, were upregulated. Immunohistochemical detection revealed that expression of CCR7 was positively associated with EMT Vimentin and Slug expression in human lung carcinoma tissues, consistent with the in vitro findings. Additionally, these results were consistent with previous studies in breast and gastric cancer cells $(15,16)$. EMT causes epithelial cells to acquire invasion and migration capacities $(1,2)$. Therefore, the CCL21/CCR7 axis not only determines the targeted migration, but also promotes cancer cell migration.

The effect of the ERK signaling pathway on CCL21/CCR7 was investigated to identify whether it activates EMT in human lung cancer cells in vitro. Following inhibition of ERK phosphorylation, invasion and migration of cancer cells were significantly decreased compared with cells without ERK inhibition. In addition, the mRNA and protein expression levels of EMT biomarkers were reduced in response to CCL21 stimulation. These results indicated that CCL21/CCR7 activates the EMT procedure by activating ERK phosphorylation. ERK1/2 belongs to the mitogen-activated protein kinase family, working as the most common signaling pathway in mediating EMT $(20,21)$. Therefore, ERK is a critical signal concerned with chemotaxis and migration of cancer cells.

It has been demonstrated by immunohistochemical staining that CCR7 is expressed in a variety of carcinomas $(4,22)$; however, its ligands CCL19 or CCL21 have rarely been investigated in previous reports. A report previously demonstrated that a melanoma cell line and several breast cancer cell lines secreted the CCR7 ligands, CCL21/19 (23), which may promote the migrating capacity of cancer cells with high levels of CCR7. However, in the present study, CCL21 was expressed at low levels in lung cancer cells, although it was expressed strongly in the endothelium of lymphatic vessels. Lymphatic vessels often exist around the cancer foci, so certain cancer cells are situated far from lymphatic vessels. Therefore, the present study aimed to identify which factor may stimulate CCR7 expression when cancer cells do not secrete CCR7 ligands. A previous study identified that, under hypoxic conditions, hypoxia-inducible factor (HIF)-1 $\alpha$ and HIF-2 $\alpha$ upregulate CCR7 expression in non-small lung cancer via the ERK1/2 signaling pathway $(24,25)$. An additional study confirmed that TGF $\beta-1$ may stimulate cancer cells to express CCR7 (26). TGF $\beta-1$, a typical cytokine for EMT stimulation, may be released widely by various cells including cancer cells in the tumor microenvironment (27). Therefore, these factors (except CCR7 ligands) may serve as more significant stimulators for CCR7 expression of cancer cells in the cancer microenvironment. This allows cancer cells rich in CCR7 to easily initiate EMT and chemotaxis processes in response to CCL21/19 released by the capillary lymphatic endothelium.

In conclusion, CCL21/CCR7 may trigger EMT via the ERK1/2 signaling pathway in human lung cancer cells. The present study provided evidence that a close interaction exists between EMT and CCL21/CCR7 chemotaxis processes in lung cancer metastasis. Targeting the signal that connects CCR7 and EMT may become an important strategy for oncotherapy. However, additional research is required regarding the network and molecular interaction of signal transduction pathways.

\section{Acknowledgements}

The present study was supported by the National Science Foundation of Shandong Province (grant no. ZR2013HM096). 


\section{References}

1. Thiery JP, Acloque H, Huang RY and Nieto MA: Epithelial-mesenchymal transitions in development and disease. Cell 139: 871-890, 2009.

2. De Craene B and Berx G: Regulatory networks defining EMT during cancer initiation and progression. Nat Rev Cancer 13: 97-110, 2013

3. Mukaida $\mathrm{N}$ and Baba T: Chemokines in tumor development and progression. Exp Cell Res 318: 95-102, 2012.

4. Zlotnik A, Burkhardt AM and Homey B: Homeostatic chemokine receptors and organ-specific metastasis. Nat Rev Immunol 11: 597-606, 2011.

5. Förster R, Davalos-Misslitz AC and Rot A: CCR7 and its ligands: Balancing immunity and tolerance. Nat Rev Immunol 8: 362-371, 2008.

6. Carlsen HS, Haraldsen G, Brandtzaeg P and Baekkevold ES: Disparate lymphoid chemokine expression in mice and men: No evidence of CCL21 synthesis by human high endothelial venules. Blood 106: 444-446, 2005.

7. Baekkevold ES, Yamanaka T, Palframan RT, Carlsen HS, Reinholt FP, von Andrian UH, Brandtzaeg P and Haraldsen G: The CCR7 ligand ELC (CCL19) is transcytosed in high endothelial venules and mediates T cell recruitment. J Exp Med 193: 1105-1112, 2001.

8. Gunn MD, Tangemann K, Tam C, Cyster JG, Rosen SD and Williams LT: A chemokine expressed in lymphoid high endothelial venules promotes the adhesion and chemotaxis of naive $\mathrm{T}$ lymphocytes. Proc Natl Acad Sci USA 95: 258-263, 1998

9. Sperveslage J, Frank S, Heneweer C, Egberts J, Schniewind B, Buchholz M, Bergmann F, Giese N, Munding J, Hahn SA, et al: Lack of CCR7 expression is rate limiting for lymphatic spread of pancreatic ductal adenocarcinoma. Int J Cancer 131: E371-E381, 2012.

10. Legler DF, Uetz-von Allmen E and Hauser MA: CCR7: Roles in cancer cell dissemination, migration and metastasis formation. Int J Biochem Cell Biol 54: 78-82, 2014.

11. Li X, Ma Q, Xu Q, Liu H, Lei J, Duan W, Bhat K, Wang F, $\mathrm{Wu}$ E and Wang Z: SDF-1/CXCR4 signaling induces pancreatic cancer cell invasion and epithelial-mesenchymal transition in vitro through non-canonical activation of Hedgehog pathway. Cancer Lett 322: 169-176, 2012.

12. Chen K, Li Z, Jiang P, Zhang X, Zhang Y, Jiang Y, He Y and Li X: Co-expression of CD133, CD44v6 and human tissue factor is associated with metastasis and poor prognosis in pancreatic carcinoma. Oncol Rep 32: 755-763, 2014.

13. Lee SH, Kang HY, Kim KS, Nam BY, Paeng J, Kim S, Li JJ, Park JT, Kim DK, Han SH, et al: The monocyte chemoattractant protein-1 (MCP-1)/CCR2 system is involved in peritoneal dialysis-related epithelial-mesenchymal transition of peritoneal mesothelial cells. Lab Invest 92: 1698-1711, 2012.

14. Marsigliante S, Vetrugno C and Muscella A: Paracrine CCL20 loop induces epithelial-mesenchymal transition in breast epithelial cells. Mol Carcinog 55: 1175-1186, 2016.
15. Li F, Zou Z, Suo N, Zhang Z, Wan F, Zhong G, Qu Y, Ntaka KS and Tian H: CCL21/CCR7 axis activating chemotaxis accompanied with epithelial-mesenchymal transition in human breast carcinoma. Med Oncol 31: 180, 2014.

16. Zhang J, Zhou Y and Yang Y: CCR7 pathway induces epithelial-mesenchymal transition through up-regulation of Snail signaling in gastric cancer. Med Oncol 32: 467, 2015.

17. Siegel RL, Miller KD and Jemal A: Cancer Statistics, 2017. CA Cancer J Clin 67: 7-30, 2017.

18. Livak KJ and Schmittgen TD: Analysis of relative gene expression data using real-time quantitative PCR and the 2(-Delta Delta C(T)) Method. Methods 25: 402-408, 2001.

19. Goldstraw P, Chansky K, Crowley J, Rami-Porta R, Asamura H, Eberhardt WE, Nicholson AG, Groome P, Mitchell A, Bolejack $\mathrm{V}$, et al: The IASLC lung cancer staging project: proposals for revision of the TNM stage groupings in the forthcoming (Eighth) edition of the TNM classification for lung cancer. J Thorac Oncol 11: 39-51, 2016.

20. Chen X, Ye S, Xiao W, Wang W, Luo L and Liu Y: ERK1/2 pathway mediates epithelial-mesenchymal transition by cross-interacting with TGF//Smad and Jagged/Notch signaling pathways in lens epithelial cells. Int J Mol Med 33: 1664-1670, 2014.

21. Buonato JM and Lazzara MJ: ERK1/2 blockade prevents epithelial-mesenchymal transition in lung cancer cells and promotes their sensitivity to EGFR inhibition. Cancer Res 74: 309-319, 2014.

22. Maekawa S, Iwasaki A, Shirakusa T, Kawakami T, Yanagisawa J, Tanaka T, Shibaguchi H, Kinugasa T, Kuroki M and Kuroki M: Association between the expression of chemokine receptors CCR7 and CXCR3, and lymph node metastatic potential in lung adenocarcinoma. Oncol Rep 19: 1461-1468, 2008.

23. Shields JD, Fleury ME, Yong C, Tomei AA, Randolph GJ and Swartz MA: Autologous chemotaxis as a mechanism of tumor cell homing to lymphatics via interstitial flow and autocrine CCR7 signaling. Cancer Cell 11: 526-538, 2007.

24. Li Y, Zhang Q, Jiang L, Qiu X and Wang E: Upregulation of the Chemokine Receptor CCR7 expression by HIF-1alpha and HIF-2alpha in non-small cell lung cancer. Zhongguo Fei Ai Za Zhi 11: 724-728, 2008 (In Chinese).

25. Li Y, Zhang Q, Wang Y, Qiu X and Wang E: Effects of hypoxia on the expression of CCR7 and proliferation, invasiveness of A549 cells. Zhongguo Fei Ai Za Zhi 11: 704-706, 2008 (In Chinese).

26. Pang MF, Georgoudaki AM, Lambut L, Johansson J, Tabor V, Hagikura K, Jin Y, Jansson M, Alexander JS, Nelson CM, et al: TGF- 31 -induced EMT promotes targeted migration of breast cancer cells through the lymphatic system by the activation of CCR7/CCL21-mediated chemotaxis. Oncogene 35: 748-760, 2016.

27. Papageorgis $\mathrm{P}:$ TGF $\beta$ signaling in tumor initiation, epithelial-to-mesenchymal transition and metastasis. J Oncol 2015: 587193, 2015. 